\title{
GRAPH HYPERSURFACES AND A DICHOTOMY IN THE GROTHENDIECK RING
}

\author{
PAOLO ALUFFI AND MATILDE MARCOLLI
}

\begin{abstract}
The subring of the Grothendieck ring of varieties generated by the graph hypersurfaces of quantum field theory maps to the monoid ring of stable birational equivalence classes of varieties. We show that the image of this map is the copy of $\mathbb{Z}$ generated by the class of a point. Thus, the span of the graph hypersurfaces in the Grothendieck ring is nearly killed by setting the Lefschetz motive $\mathbb{L}$ to zero, while it is known that graph hypersurfaces generate the Grothendieck ring over a localization of $\mathbb{Z}[\mathbb{L}]$ in which $\mathbb{L}$ becomes invertible. In particular, this shows that the graph hypersurfaces do not generate the Grothendieck ring prior to localization.

The same result yields some information on the mixed Hodge structures of graph hypersurfaces, in the form of a constraint on the terms in their Deligne-Hodge polynomials.
\end{abstract}

\section{INTRODUCTION}

The interplay between perturbative quantum field theory and the theory of motives of algebraic varieties has been extensively studied in recent years, in particular in terms of the algebro-geometric and motivic properties of the graph hypersurfaces associated to Feynman graphs of scalar quantum field theories.

In particular, one of the central results in the field is the main theorem of [5], which shows that graph hypersurfaces can be arbitrarily complicated from the motivic viewpoint: their affine complements are, 'from the standpoint of their zeta functions, the most general schemes possible' ([5], p. 149). In rough terms, this is proven by showing that graph hypersurfaces generate the Grothendieck ring $K$ (Var) of varieties. More precisely (Theorem 0.6 in [5]), graph hypersurfaces generate $S^{-1} K$ (Var) as a module over the ring $S^{-1} \mathbb{Z}[\mathbb{L}]$, where $S$ is the (saturated) multiplicative subset of $\mathbb{Z}[\mathbb{L}]$ generated by $\mathbb{L}^{n}-\mathbb{L}$ for $n>1$, with $\mathbb{L}=\left[\mathbb{A}^{1}\right]$ the Lefschetz-Tate motive.

The main result of this note will imply that this localization is in fact necessary to the result of [5]. We will show that graph hypersurfaces do not generate the Grothendieck ring as a module over $\mathbb{Z}[\mathbb{L}]$, and in fact they do not even generate the localization $S^{\prime-1} K\left(\right.$ Var) as a module over $S^{\prime-1} \mathbb{Z}[\mathbb{L}]$, where $S^{\prime}$ is generated by $\mathbb{L}^{n}-1$ for $n>0$. For example, we will show that the class of an elliptic curve is not in the span of the graph hypersurfaces if coefficients are taken in $S^{\prime-1} \mathbb{Z}[\mathbb{L}]$. As $S^{-1} \mathbb{Z}[\mathbb{L}]$ is the localization of this 
latter ring at $\mathbb{L}$, it appears that localization at $\mathbb{L}$ is crucial the result of [5]. In view of this observation, we propose the following

Question. Do graph hypersurfaces generate the localized Grothendieck ring as a module over $\mathbb{Z}\left[\mathbb{L}, \mathbb{L}^{-1}\right]$ ?

This situation illustrates a sharp dichotomy in the behavior of graph hypersurfaces in the Grothendieck ring under the contrasting operations of inverting $\mathbb{L}$ and setting $\mathbb{L}$ to zero. Graph hypersurfaces are 'as general as possible' after localization (at $\mathbb{L}$ and $S^{\prime}$ ), while they are extremely special with respect to taking the quotient modulo the ideal $(\mathbb{L})$ : as we will show, their span agrees with the span of a point modulo $(\mathbb{L})$.

We quickly recall some basic notation and terminology. For a connected finite graph $G$ with $n$ edges the graph polynomial $\psi_{G}\left(t_{1}, \ldots, t_{n}\right)$ is defined as

$$
\psi_{G}\left(t_{1}, \ldots, t_{n}\right)=\sum_{T \subseteq G} \prod_{e \notin T} t_{e}
$$

where $T$ runs over the spanning trees of $G$ and $t_{e}$ is the variable associated to an edge $e$. In general, we define the graph polynomial for a (finite) graph $G$ to be the product of the polynomials for the connected components of $G$. We denote by $X_{G}$ the projective hypersurface defined by the homogeneous polynomial $\psi_{G}$ in $\mathbb{P}^{n-1}$, by $\widehat{X}_{G} \subset \mathbb{A}^{n}$ the affine hypersurface, and by $Y_{G} \subseteq$ $\mathbb{A}^{n}$ the affine hypersurface complement $Y_{G}=\mathbb{A}^{n} \backslash \widehat{X}_{G}$. As the main results of [5] are expressed in terms of $Y_{G}$, we choose to focus on $Y_{G}$ in this paper. We note that graph hypersurfaces are usually singular; also, it is easy to see that the irreducible components of $X_{G}$ are rational.

Our main result can be stated as follows. Larsen and Lunts associate with each variety $V$ (possibly singular, possibly non-compact) an element in the monoid ring $\mathbb{Z}[S B]$ generated by stable birational equivalence classes of varieties. This assignment is compatible with the relations defining $K$ (Var), and associates with $V$ its own stable birational equivalence class if $V$ is smooth and projective. Smooth projective rational varieties have class 1 in $\mathbb{Z}[S B]$, but note that the element in $\mathbb{Z}[S B]$ determined by the image of the class $[V] \in K$ (Var) of a quasi-projective or singular rational variety need not be in the 'constant' part $\mathbb{Z} \subseteq \mathbb{Z}[S B]$ in general (cf. Example 2.4). Thus, although irreducible graph hypersurfaces are rational, this fact alone does not give information on their image in $\mathbb{Z}[S B]$. What we show is precisely that the Larsen-Lunts image of graph hypersurfaces do lie in the constant part of $\mathbb{Z}[S B]$.

Theorem 1.1. Affine graph hypersurface complements span $\mathbb{Z} \subseteq \mathbb{Z}[S B]$.

Morally, Theorem 1.1 shows that graph hypersurfaces and their complements are rational in a very strong sense (which we will make precise in $\oint_{2}$ ): for example, the image in $\mathbb{Z}[S B]$ of the class of an irreducible graph hypersurface does equal the class of a point (Corollary [3.3). This is the reason why they do not span the unlocalized Grothendieck ring of varieties. 
In more classical terms, Theorem 1.1 provides some information on the mixed Hodge structure of graph hypersurfaces, as it shows that the DeligneHodge polynomial of a graph hypersurface is necessarily of the form $c+$ $u v P(u, v)$, with $c \in \mathbb{Z}$. This shows that elliptic curves are not in the span of graph hypersurfaces in the Grothendieck ring.

Theorem 1.1 is proven by using the realization of $\mathbb{Z}[S B]$ as the quotient $K(\operatorname{Var}) /(\mathbb{L})$ (also recalled in 92 ). An explicit computation (Theorem 3.2) based on a deletion-contraction formula for the Grothendieck class of a graph hypersurface shows that for every graph $G$, the class of $Y_{G}$ modulo $(\mathbb{L})$ is 0 or \pm 1 . Theorem 1.1 and the consequences mentioned above follow immediately.

\section{Stable Birational equivalence and the Grothendieck Ring}

In the following, we denote by $K$ (Var) the Grothendieck ring of varieties. This is generated by the isomorphism classes of irreducible quasi-projective varieties with the inclusion-exclusion relations $[X]=[X \backslash Y]+[Y]$, for closed embeddings $Y \subseteq X$, and with the product $[X] \cdot[Y]=[X \times Y]$. The Grothendieck ring $K$ (Var) depends on the field of definition of the varieties. This will be understood to be $\mathbb{Q}$ in the following.

A result of [10] relates the Grothendieck ring to the ring of stable birational equivalence classes of varieties. We comment here briefly on some aspects of this result that will be useful in the discussion of the case of graph hypersurfaces.

Two (irreducible, complex) varieties $X, Y$ are stably birational if $X \times \mathbb{P}^{k}$ is birational to $Y \times \mathbb{P}^{\ell}$ for some $k, \ell \geq 0$. If $X$ is stably birational to $Y$, and $X^{\prime}$ is stably birational to $Y^{\prime}$, then $X \times X^{\prime}$ is stably birational to $Y \times Y^{\prime}$. Thus, the set of classes of stable birational equivalence of varieties is a multiplicative monoid $S B$, with unit equal to the class of a point.

Theorem 2.1. ([10], Proposition 2.7.) Let $(\mathbb{L})$ be the ideal in $K(\operatorname{Var})$ generated by the Lefschetz motive $\mathbb{L}=\left[\mathbb{A}^{1}\right]$. The ring $K(\operatorname{Var}) /(\mathbb{L})$ is isomorphic to the monoid ring $\mathbb{Z}[S B]$.

This result is obtained by defining a homomorphism $K(\operatorname{Var}) \rightarrow \mathbb{Z}[S B]$, sending $[V]$ to the stable birational equivalence class $[V]_{S B}$ of $V$ for every irreducible smooth, projective variety $V$. The main technical step is to show that this homomorphism is well-defined; this may be proven by using Bittner's alternative description (4]) of the Grothendieck ring of varieties $K$ (Var) with generators that are smooth projective varieties and relations

$$
[X]-[Y]=\left[B \ell_{Y}(X)\right]-[E],
$$

for a smooth closed subvariety $Y \subseteq X$, with $B \ell_{Y}(X)$ the blowup of $X$ along $Y$ and $E$ the exceptional divisor. This relation replaces the usual inclusion-exclusion relation $[X]=[X \backslash Y]+[Y]$, which requires the noncompact $X \backslash Y$. Bittner's characterization depends on the weak factorization theorem of [1], which shows that any proper birational map between smooth 
irreducible varieties over a field of characteristic zero can be factored into a sequence of blow-ups and blow-downs with smooth centers.

Theorem 2.1 is stated over $\mathbb{C}$ in $[10$, but holds over $\mathbb{Q}$ as well since so does the weak factorization theorem (Remark 2 after Theorem 0.3 .1 of [1]); this is also observed explicitly in [9], p. 28.

Remark 2.2. Stable birational equivalence makes sense for every variety $V$, so every variety (whether or not smooth and projective) has a class $[V]_{S B}$ in $\mathbb{Z}[S B]$. It is important to keep in mind that in general this class agrees with the image of $[V] \in K($ Var) via the Larsen-Lunts homomorphism only if $V$ is smooth and projective. In other cases the image of $[V]$ in $\mathbb{Z}[S B]$ may be determined by expressing $[V]$ as a combination of classes of smooth projective varieties, and then reproducing that combination in $\mathbb{Z}[S B]$.

For example, the image of $\mathbb{L}=\left[\mathbb{A}^{1}\right]$ is 0 in $\mathbb{Z}[S B]$ because $\left[\mathbb{A}^{1}\right]=\left[\mathbb{P}^{1}\right]-$ $\left[\mathbb{P}^{0}\right]$ in $K($ Var $)$, and $\mathbb{P}^{0}, \mathbb{P}^{1}$ are trivially stably birationally equivalent to each other. Likewise, the image of (the class of) an irreducible nodal plane cubic $C$ in $\mathbb{Z}[S B]$ is $0 \neq 1$ even though $[C]_{S B}=\left[\mathbb{P}^{0}\right]_{S B}$, since $[C]=\left[\mathbb{P}^{1}\right]-\left[\mathbb{P}^{0}\right]$ in $K$ (Var).

To make this point more explicit, we introduce a notion of ' $\mathbb{L}$-equivalence'.

Definition 2.3. Two irreducible quasi-projective varieties $X, Y$ are $\mathbb{L}$ equivalent if their classes in $\mathbb{Z}[S B]$ via the Larsen-Lunts isomorphism coincide, that is, if $[X] \equiv[Y] \bmod (\mathbb{L})$ in $K(\operatorname{Var})$. A variety is $\mathbb{L}$-rational if it is $\mathbb{L}$-equivalent to $\mathbb{P}^{k}$, for some $k \geq 0$.

If $X$ and $Y$ are irreducible smooth projective and stably birational, then they are also $\mathbb{L}$-equivalent; however, this is not necessarily the case if $X$, $Y$ are not smooth and/or not complete. For example, as observed above, an irreducible nodal cubic in $\mathbb{P}^{2}$ is complete and birational to $\mathbb{P}^{1}$ but not $\mathbb{L}$-rational.

In fact, the following example shows that rational (singular, projective) varieties may be very far from being $\mathbb{L}$-rational.

Example 2.4. There exists a complete rational surface $X$ whose LarsenLunts image in $\mathbb{Z}[S B]$ is $2-[C]_{S B}$, where $C$ is an elliptic curve.

Indeed, by Theorem 3.3 of [8], there exist projective rational surfaces $X$ with one isolated singular point $p$ such that the exceptional divisor in the minimal resolution $\widetilde{X}$ of $X$ is an elliptic curve $C$. Using Bittner's relations, $[X]=[\tilde{X}]-[C]+[p]$ in $K(\operatorname{Var})$, and since all varieties on the right-hand side are smooth and projective, and $\widetilde{X}$ is rational, then the image of $[X]$ in $\mathbb{Z}[S B]$ equals $[\tilde{X}]_{S B}-[C]_{S B}+[p]_{S B}=2-[C]_{S B}$.

These caveats apply to graph hypersurfaces. As recalled in the introduction, irreducible (projective) graph hypersurfaces $X_{G}$ are easily seen to be rational, and are complete, but are in general singular. Affine graph hypersurface complements $Y_{G}$ are trivially rational, but non-complete. As 
observed above, these naive considerations do not suffice to determine the Larsen-Lunts images of these varieties in $\mathbb{Z}[S B]$.

\section{Graph hypersurfaces and stable birational EQUiVALENCE}

In [3], we proved a deletion-contraction formula for the classes in the Grothendieck ring of the graph hypersurfaces. We recall here the result, for completeness, since the conclusion we derive on the stable birational equivalence classes will be a direct consequence of this formula. We also note that the result is also implicit in the literature preceding [3], e.g. [11], 6].

Theorem 3.1. ([3], Theorem 3.8) Let $G$ be a graph with $n$ edges, and let $e$ be an edge of $G$. Denote by $G \backslash e$ the graph obtained by removing e, and by $G /$ e the graph obtained by contracting e. Let $Y_{G}$ denote the affine graph hypersurface complement in $\mathbb{A}^{n}$.

- If e is a bridge in $G$, then $\left[Y_{G}\right]=\mathbb{L} \cdot\left[Y_{G \backslash e}\right]$;

- If $e$ is a looping edge in $G$, then $\left[Y_{G}\right]=(\mathbb{L}-1) \cdot\left[Y_{G \backslash e}\right]$;

- If e is a neither a bridge nor a looping edge in $G$, then

$$
\left[Y_{G}\right]=\mathbb{L} \cdot\left[\mathbb{A}^{n-1} \backslash Z\right]-\left[Y_{G \backslash e}\right],
$$

where $Z$ is the intersection of the affine graph hypersurfaces for $G \backslash e$, $G / e$ in $\mathbb{A}^{n-1}$.

Proof. We give a quick proof for completeness; for more details, see [3]. Let $t_{i}$ be the variable corresponding to the $i$-th edge $e_{i}$, and assume that $e=e_{n}$ is not a bridge or a looping edge. In terms of graph polynomials:

$$
P_{G}\left(t_{1}, \ldots, t_{n}\right)=t_{n} P_{G \backslash e}\left(t_{1}, \ldots, t_{n-1}\right)+P_{G / e}\left(t_{1}, \ldots, t_{n-1}\right) .
$$

The complement $Y_{G}$ is the set of $n$-tuples $\left(t_{1}, \ldots, t_{n}\right)$ such that $P_{G}\left(t_{1}, \ldots, t_{n}\right)$ does not vanish; thus, such that

$$
t_{n} P_{G \backslash e}\left(t_{1}, \ldots, t_{n-1}\right) \neq-P_{G / e}\left(t_{1}, \ldots, t_{n-1}\right) .
$$

Over $Y_{G \backslash e}$ (that is, if $P_{G \backslash e} \neq 0$ ), this condition holds if and only if $t_{n} \neq$ $-P_{G / e} / P_{G \backslash e}$, that is, for $t_{n} \in \mathbb{A}^{1} \backslash \mathbb{A}^{0}$. Over the rest of the complement of $Z$ (that is, if $P_{G \backslash e} \neq 0$ but $P_{G / e}=0$ ), the condition is satisfied for all $t_{n}$, hence for $t_{n} \in \mathbb{A}^{1}$. Over $Z$ (that is, if $P_{G \backslash e}=P_{G / e}=0$ ), the condition is not satisfied for any choice of $t_{n}$. Therefore,

$$
\left[Y_{G}\right]=(\mathbb{L}-1) \cdot\left[Y_{G \backslash e}\right]+\mathbb{L} \cdot\left[\left(\mathbb{A}^{n-1} \backslash Z\right) \backslash Y_{G \backslash e}\right],
$$

and this is equivalent to third equality stated above. The other cases are analogous.

The deletion-contraction formula yields the following computation of the Larsen-Lunts image of $Y_{G}$ in $\mathbb{Z}[S B] \cong K($ Var $) /(\mathbb{L})$.

\section{Theorem 3.2.}

$\left[Y_{G}\right] \equiv\left\{\begin{array}{ccl}0 & \bmod (\mathbb{L}) & \text { if } G \text { has edges that are not looping edges } \\ (-1)^{n} & \bmod (\mathbb{L}) \quad \text { if } G \text { has } n \geq 0 \text { looping edges, and no other edge. }\end{array}\right.$ 
Proof. Reading the result of Theorem 3.1 above modulo $(\mathbb{L})$ gives:

- If $e$ is a bridge in $G$, then $\left[Y_{G}\right]=0 \bmod (\mathbb{L})$;

- If $e$ is not a bridge $G$, then $\left[Y_{G}\right]=-\left[Y_{G \backslash e}\right] \bmod (\mathbb{L})$.

If now $G$ has any edge that is not a looping edge, then removing all but one such edge leaves a graph with a bridge, and hence $\left[Y_{G}\right] \equiv 0 \bmod (\mathbb{L})$ in this case. If all $n$ edges of $G$ are looping edges, then repeated application of the second formula shows that $\left[Y_{G}\right] \equiv(-1)^{n} \cdot\left[Y_{G}\right] \bmod (\mathbb{L})$, where $\underline{G}$ is the graph obtained by removing all edges from $G$. Clearly $Y_{G}=\mathbb{A}^{0}$, so $\left[Y_{G}\right] \equiv(-1)^{n} \bmod (\mathbb{L})$ in this case, as stated.

In terms of projective graph hypersurfaces:

Corollary 3.3. Let $G$ be a graph that is not a forest, and with at least one non-looping edge. Then the projective graph hypersurface $X_{G}$ is $\mathbb{L}$-rational.

Proof. Note that $G$ must have at least 2 edges. Since $G$ is not a forest, $\widehat{X}_{G}$ is not empty; and by Theorem 3.2, since $G$ has non-looping edges, then the class $\left[Y_{G}\right]$ is in the ideal $(\mathbb{L})$. The affine complement $Y_{G}=\mathbb{A}^{n} \backslash \widehat{X}_{G}$ fibers over the projective complement $\mathbb{P}^{n-1} \backslash X_{G}$, with fibers $\mathbb{A}^{1} \backslash \mathbb{A}^{0}$. Therefore

$$
(\mathbb{L}-1) \cdot\left[\mathbb{P}^{n-1} \backslash X_{G}\right] \in(\mathbb{L}),
$$

and hence $\left[\mathbb{P}^{n-1} \backslash X_{G}\right] \in(\mathbb{L})$; thus

$$
\left[X_{G}\right] \equiv\left[\mathbb{P}^{n-1}\right] \bmod (\mathbb{L}),
$$

showing that $X_{G}$ is $\mathbb{L}$-equivalent to $\mathbb{P}^{n-1}$.

Again we remark that if $X_{G}$ is irreducible, then it is easily seen to be rational, but examples such as Example 2.4 show that this does not suffice in itself to draw the conclusion stated in Corollary 3.3

In view of the result of [10] recalled in Theorem 2.1 above, Theorem 3.2 has the following immediate consequence:

Corollary 3.4. Let $\mathcal{R}$ be the subring of $K(\operatorname{Var})$ spanned by the classes $\left[Y_{G}\right]$. Then the image of $\mathcal{R}$ in $\mathbb{Z}[S B]$ via the Larsen-Lunts homomorphism is the subring $\mathbb{Z}$ generated by the stable birational equivalence class of a point.

Proof. The image of $\mathcal{R}$ in $\mathbb{Z}[S B]$ is the quotient $\mathcal{R} /(\mathbb{L})$. By Theorem 3.2 , $\mathcal{R} /(\mathbb{L}) \cong \mathbb{Z}$.

Remark 3.5. With our conventions, the product of two classes $\left[Y_{G_{1}}\right],\left[Y_{G_{2}}\right]$ is itself the class $\left[Y_{G_{1} \amalg G_{2}}\right]$ of the affine complement of a graph hypersurface (the class of the affine hypersurface complement is a 'motivic Feynman rule', see Proposition 2.5 in [2]). Further, the Lefschetz motive $\mathbb{L}$ equals $\left[Y_{G}\right]$ for the graph $G$ consisting of a single edge joining two distinct vertices. Thus, the ring $\mathcal{R}$ generated by the classes $\left[Y_{G}\right]$ agrees with the $\mathbb{Z}[\mathbb{L}]$-module generated by the classes $\left[Y_{G}\right]$. Therefore, the following immediate consequence of Corollary 3.4 formalizes the first 'non-spanning' result mentioned in the Introduction. 
Theorem 3.6. The classes $\left[Y_{G}\right]$ of the affine graph hypersurface complements do not span the Grothendieck ring $K($ Var) over $\mathbb{Z}[\mathbb{L}]$.

Proof. In view of Corollary [3.4, it suffices to notice that $\mathbb{Z}[S B] \neq \mathbb{Z}$. This is clear since there are polynomial invariants of smooth projective varieties that are invariant under stable birational equivalence, and are not always constant. One example is given in [10, Definition 3.4 in terms of Hodge polynomials.

As mentioned in the Introduction, this observation can be sharpened, in a way that relates well to the result of [5].

Corollary 3.7. Let $S^{\prime}$ be the saturated multiplicative subset of $\mathbb{Z}[\mathbb{L}]$ generated by the elements $\mathbb{L}^{n}-1$, for $n>0$. Then the classes $\left[Y_{G}\right]$ do not generate $S^{\prime-1} K($ Var $)$ over $S^{\prime-1} \mathbb{Z}[\mathbb{L}]$.

Proof. Localization commutes with taking quotients: the quotient

$$
\left(S^{\prime-1} K(\operatorname{Var})\right) /(\mathbb{L})
$$

equals the localization $S^{\prime-1}(K(\operatorname{Var}) /(\mathbb{L}))$. Since all elements of $S^{\prime}$ are invertible modulo $(\mathbb{L})$, this latter equals $K(\operatorname{Var}) /(\mathbb{L})$; and the action of $\mathbb{L}$ on this module is 0 . Since the classes $\left[Y_{G}\right]$ do not span this quotient, they cannot span $S^{\prime-1} K($ Var $)$ over $S^{\prime-1} \mathbb{Z}[\mathbb{L}]$.

Corollary 3.7 should be compared with Theorem 0.6 in [5], which states that the classes $\left[Y_{G}\right] d o$ generate the localization $S^{-1} K\left(\operatorname{Var}_{\mathbb{Z}}\right)$ over $S^{-1} \mathbb{Z}[\mathbb{L}]$, where $S$ is the multiplicative system generated by $\mathbb{L}^{n}-\mathbb{L}$ for $n>1$. A fortiori, the classes $\left[Y_{G}\right]$ generate $S^{-1} K\left(\operatorname{Var}_{\mathbb{Q}}\right)$. Localizing at $S$ amounts to localizing at $S^{\prime}$ and at $\mathbb{L}$; Corollary 3.7 shows that the localization at $\mathbb{L}$ is crucial to the mentioned result in [5]. This suggests that the classes $\left[Y_{G}\right]$ may possibly span the Grothendieck ring over the simpler localization $\mathbb{Z}\left[\mathbb{L}^{-1}, \mathbb{L}\right]$.

We end by observing that the results stated above have a straightforward Hodge-theoretic formulation. Every smooth complex projective variety $X$ carries Hodge numbers $h^{p, q}(X)$. The Hodge polynomial of $X$ is the polynomial $\sum_{p, q}(-1)^{p+q} h^{p, q}(X) u^{p} v^{q}$. Now, the Hodge polynomial determines a ring homomorphism $K$ (Var) $\rightarrow \mathbb{Z}[u, v]$, see e.g. $\S 2.11$ in [12]; this homomorphism maps $\mathbb{L}$ to $u v$. Indeed, the Hodge polynomial may be consistently defined for all varieties and satisfies the relations in $K$ (Var), as observed in [7. This ('Deligne-Hodge') polynomial of an arbitrary complex variety $X$ records information about the mixed Hodge structure on the cohomology $H_{c}^{k}(X, \mathbb{Q})$ of $X$ with compact support: it may be defined as $\sum_{p, q} e^{p, q}(X) u^{p} v^{q}$, where

$$
e^{p, q}(X)=\sum_{k}(-1)^{k} h^{p, q}\left(H_{c}^{k}(X, \mathbb{Q})\right) .
$$

Corollary 3.8. Let $X$ be any complex projective variety whose class $[X]$ is in the subring $\mathcal{R}$ of $K\left(\operatorname{Var}_{\mathbb{C}}\right)$ generated by the classes $\left[Y_{G}\right]$, as $G$ ranges over 
all graphs. Then the Deligne-Hodge polynomial of $X$ is of the form

$$
c+u v P(u, v)
$$

with $c \in \mathbb{Z}$.

Proof. The Hodge polynomial induces a homomorphism

$$
K(\operatorname{Var}) /(\mathbb{L}) \rightarrow \mathbb{Z}[u, v] /(u v) .
$$

The image of $\mathcal{R} /(\mathbb{L}) \cong \mathbb{Z}$ in $\mathbb{Z}[u, v] /(u v)$ is $\mathbb{Z}$, and the statement follows.

For example, $h^{1,0}(X) \neq 0$ for e.g., a smooth elliptic curve. Therefore, Corollary 3.8 shows that classes of elliptic curves are not in the span of the classes $\left[Y_{G}\right]$.

\section{REFERENCES}

[1] D. Abramovich, K. Karu, K. Matsuki, J. Wlodarczyk, Torification and factorization of birational maps, J. Amer. Math. Soc. 15 (2002), 531-572.

[2] P. Aluffi, M. Marcolli, Algebro-geometric Feynman rules, arXiv:0811.2514.

[3] P. Aluffi, M. Marcolli, Feynman motives and deletion-contraction relations, arXiv:0907.3225.

[4] F. Bittner, The universal Euler characteristic for varieties of characteristic zero. Compos. Math. 140 (2004), no. 4, 1011-1032.

[5] P. Belkale, P. Brosnan, Matroids, motives, and a conjecture of Kontsevich, Duke Math. Journal, Vol.116 (2003) 147-188.

[6] Spencer Bloch, Hélène Esnault, and Dirk Kreimer. On motives associated to graph polynomials. Comm. Math. Phys., 267(1):181-225, 2006.

[7] V.I. Danilov and A.G. Khovanskiǔ, Newton polyhedra and an algorithm for calculating Hodge-Deligne numbers, Izv. Akad. Nauk SSSR Ser. Mat., Vol.50 (1986) N.5, 925945.

[8] S. Greco, A. Vistoli, On the construction of rational surfaces with assigned singularities, in "Algebraic geometry - open problems (Ravello, 1982)", Lecture Notes in Math., Vol. 997, pp.210-217, Springer, 1983.

[9] J. Kollár, Conics in the Grothendieck ring. Adv. Math. 198 (2005), no. 1, 27-35.

[10] M. Larsen, V.A. Lunts, Motivic measures and stable birational geometry. Mosc. Math. J. 3 (2003), no. 1, 85-95, 259.

[11] John R. Stembridge. Counting points on varieties over finite fields related to a conjecture of Kontsevich. Ann. Comb., 2(4):365-385, 1998.

[12] W. Veys, Arc spaces, motivic integration and stringy invariants, in "Singularity theory and its applications", Volume 43, Adv. Stud. Pure Math., pp. 529-572. Math. Soc. Japan, 2006.

Department of Mathematics, Florida State University, Tallahassee, FL 32306, USA

E-mail address: aluffi@math.fsu.edu

Department of Mathematics, California Institute of Technology, Pasadena, CA 91125, USA

E-mail address: matilde@caltech.edu 